\author{
Ann L. Collins - Deqiong Ma - Patrice L. Whitehead \\ Eden R. Martin · Harry H. Wright • \\ Ruth K. Abramson · John P. Hussman • \\ Jonathan L. Haines • Michael L. Cuccaro • \\ John R. Gilbert · Margaret A. Pericak-Vance
}

\title{
Investigation of autism and GABA receptor subunit genes in multiple ethnic groups
}

Received: 2 February 2006 / Accepted: 13 April 2006 / Published online: 13 June 2006

(C) Springer-Verlag 2006

\begin{abstract}
Autism is a neurodevelopmental disorder of complex genetics, characterized by impairment in social interaction and communication, as well as repetitive behavior. Multiple lines of evidence, including alterations in levels of GABA and GABA receptors in autistic patients, indicate that the GABAergic system, which is responsible for synaptic inhibition in the adult brain, may be involved in autism. Previous studies in our lab indicated association of noncoding single nucleotide polymorphisms (SNPs) within a GABA receptor subunit gene on chromosome 4, GABRA4, and interaction between SNPs in GABRA4 and GABRB1 (also on chromosome 4), within Caucasian autism patients. Studies of genetic variation in African-American autism families are rare. Analysis of 557 Caucasian and an independent population of 54 AfricanAmerican families with 35 SNPs within GABRB1 and GABRA4 strengthened the evidence for involvement of GABRA4 in autism risk in Caucasians (rs17599165, $p=0.0015 ; \quad$ rs1912960, $p=0.0073 ;$ and rs17599416, $p=0.0040)$ and gave evidence of significant association in African-Americans (rs2280073, $p=0.0287$ and rs168 59788, $p=0.0253$ ). The GABRA4 and GABRB1 interaction was also confirmed in the Caucasian dataset (most
\end{abstract}

A. L. Collins · D. Ma · P. L. Whitehead · E. R. Martin

M. L. Cuccaro · J. R. Gilbert · M. A. Pericak-Vance $(\square)$

Center for Human Genetics, Duke University Medical Center,

Durham, NC, USA

e-mail: mpv@chg.mc.duke.edu

Tel.: +1-919-6842063

Fax: +1-919-6840910

H. H. Wright · R. K. Abramson

School of Medicine, University of South Carolina,

Columbia, SC, USA

\section{J. P. Hussman}

Hussman Foundation,

Ellicott City, MD, USA

\section{J. L. Haines}

The Center for Human Genetics Research,

Vanderbilt University Medical Center,

Nashville, TN, USA significant pair, rs1912960 and rs2351299; $p=0.004$ ). Analysis of the subset of families with a positive history of seizure activity in at least one autism patient revealed no association to GABRA4; however, three SNPs within GABRB1 showed significant allelic association; rs2351 $299(p=0.0163), \operatorname{rs} 4482737(p=0.0339)$, and $\mathrm{rs} 3832300$ $(p=0.0253)$. These results confirmed our earlier findings, indicating GABRA4 and GABRB1 as genes contributing to autism susceptibility, extending the effect to multiple ethnic groups and suggesting seizures as a stratifying phenotype.

Keywords Autism - SNPs - GABA receptors ·

Association · Ethnicity

\section{Introduction}

Autism is a neurodevelopmental disorder characterized by severe impairment in social interaction and communication, as well as repetitive behavior. Autism is part of a spectrum of disorders denoted autism spectrum disorders (ASD), which in addition to autism, include Asperger syndrome, childhood disintegrative disorder, and pervasive developmental disorder not otherwise specified (PDD-NOS). Onset of this disorder is typically before 3 years of age. The incidence of autism is estimated at approximately one in 1,000 individuals, with an increased incidence in males [1-3]. Incidences of approximately two to three in 1,000 are reported when a broader diagnosis includes the entire spectrum of pervasive developmental disorders [2, 4].

Evidence indicates that this disease has a strong genetic component. Twin studies have shown that monozygote twins have a higher recurrence rate than dizygotic twins. While the actual percentages have varied, recurrence rates have been measured as high as $95 \%$ in monozygote twins and $23 \%$ in dizygotic twins [5-7]. Despite this strong evidence for a genetic component, the inheritance appears to be complex, with estimates that more than 15 genes may be involved in its inheritance $[8,9]$, with different families carrying different combinations of contributing genes. The 
genes may act independently or interactively, adding to the complexity of determining the genetic contribution to this disorder.

There are two approaches to identifying genetic contributors to disease. The first is a genome wide search in which linkage or association analysis is used to identify regions of the genome that may contain autism susceptibility genes. The second is the candidate gene approach, which investigates a specific gene or genes for involvement in autism risk. In the candidate gene approach, genes are chosen for study based on either what is known about the gene's function, its location (for example in a recognized linkage peak), or a combination of both. Several candidates are hypothesized to be involved in autism; however, no single candidate gene has consistently emerged as involved in autism risk.

One candidate pathway that is hypothesized to be involved in autism is the GABAergic system. Hussman [10] suggested that autism is the result of an imbalance of the excitatory glutamatergic and inhibitory GABAergic pathways, resulting in overstimulation in the brain and inability to filter out excess stimuli from environmental and intrinsic sources. This theory is supported by multiple lines of evidence. First, histological, biochemical, and molecular approaches have demonstrated altered levels and distribution of GABA and GABA receptors in peripheral blood and plasma, as well as in the brain, including decreased GABA-A receptors and benzodiazepine binding sites in the hippocampal formation [11-13]. There are also reported alterations in GABAergic neurons, as demonstrated by the increased packing density of GABAergic interneurons in the CA3 and CA1 subfields, and by the decreased numbers and reduced size of cerebellar GABAergic Purkinje cells $[14,15]$. In addition, duplications and isodicentric chromosomes in the region containing the three clustered GABA receptor subunits GABRB3, GABRA5, and GABRG3 on chromosome $15 \mathrm{q}$ have been associated with autism $[16,17]$. As well, evidence for both linkage and allelic association have been reported for this same GABA gene cluster, although the findings have not been consistent across datasets [18-22]. Investigation of association of GABA receptor subunits outside of the chromosome 15 region has been limited [23]. Lastly, mutations have been reported in multiple GABA receptor genes in families with epilepsy [24]. Given the high comorbidity of autism with epilepsy and seizures, these data suggest that a similar molecular etiology could exist between the disorders.

Signaling in the GABAergic system is mediated by receptors for the neurotransmitter GABA. There are 19 known GABA receptor subunits arranged in clusters throughout the genome. Functional pentamers formed by various combinations of these subunits result in receptors of varying properties and sensitivities. The amounts and functional capabilities of individual receptor subunits that form a specific pentamer can affect the amount and quality of signaling in different parts of the brain.

Previously published work from our laboratory analyzed 14 autosomal GABA receptor genes on four different chromosomes, using 70 SNPs in a Caucasian dataset of 470 families. This analysis revealed allelic association to rs1912960 $(p=0.01)$ within gamma-aminobutyric acid (GABA) A receptor, alpha-4 (GABRA4) on chromosome 4 [23] and a significant interaction with rs 2351299 within the neighboring gene gamma-aminobutyric acid (GABA) A receptor, beta-1 (GABRB1).

Despite similar prevalence rates between Caucasian and African-Americans [25, 26], autism studies in AfricanAmericans are rare. Risk alleles may be different between ethnic groups or the same risk alleles may have differential effects in each ethnic group, warranting studies in multiple groups. Evidence that phenotypic factors, including indicators of language development, may be more severe in African-Americans, compared to non-Hispanic Caucasians, [27] is consistent with these possibilities and underscores the need to investigate autism in different ethnic groups. In this study, we present a validation of our previous report in an independent dataset of 54 African-American families, as well as confirmation of our previous results in an expanded Caucasian sample of 557 autism families.

\section{Materials and methods}

\section{Samples}

All families were drawn from a large multisite study of autism genetics conducted in the southeastern United States. These families are recruited through the Center for Human Genetics (CHG) at Duke University Medical Center (DUMC), the University of South Carolina, and the Center for Human Genetic Research at Vanderbilt ( $N=54$ African-American and 557 Non-Hispanic Caucasian families) through support groups, advertisements, and clinical and educational settings. All sites recruited, enrolled, and sampled individuals with autism and family members, per study protocols approved by their respective institutional review boards (IRBs). Written informed consent was obtained from parents and from children who were able to give informed consent. Families were enrolled based on probands meeting the following core inclusion criteria of: (1) probands ranging from three to 21 years of age; (2) a presumptive clinical diagnosis of autism; and (3) an expert clinical diagnosis of autism using DSM-IV criteria [28], supported by the Autism Diagnostic Interview-Revised (ADI-R) [29] and in some cases, the Autism Diagnostic Observation Schedule (ADOS) [30]. Our original ascertainment protocol relied on clinical expertise and the ADI-R. The ADOS was subsequently added as a required diagnostic method, and families that were missing ADOS assessments were updated as available. To assure valid ADI-R results, all participants who met current diagnostic criteria for autism were included only if they had a minimal developmental level of 18 months, as extrapolated from the Vineland Adaptive Behavior Scale score [31], or had an IQ equivalent greater than 35 based on scores from a standardized measure of cognitive ability such as an the Wechsler Scale of Intelligence for Children - 4th edition [32], Differential 
Abilities Scale [33], Mullen Scales of Early Learning [34] or Leiter Intelligence Scale-Revised [35]. IQ data was derived from medical records or direct assessment. Exclusion criteria for participation in the larger genetics study included: severe sensory problems (e.g., visual impairment or hearing loss), significant motor impairments (e.g., failure to sit by 12 months, or walk by 24 months), or identified metabolic, genetic, or progressive neurological disorders, based on screening by clinical staff. Additional samples are from the Autism Genetic Research Exchange (AGRE). These individuals were qualified using similar methods including ADI-R, ADOS, VABS, and a standardized measure of IQ.

Thirty-nine African-American families were used in an initial GABA receptor screen. Follow-up analysis of significant findings was performed in 54 African-American families. Analysis of the extended Caucasian dataset included 557 non-Hispanic Caucasian families. Onehundred and five new non-Hispanic Caucasian families were added to the analysis ( 18 families previously analyzed by Ma et al. [23] were newly identified as Hispanic, and were omitted from the current study in an effort decrease heterogeneity in the Caucasian dataset).

Classification of history of seizure activity in autism patients was based on question 92 from the ADI-R, which queries for both current and lifetime presence of convulsions, seizures, and epilepsy. Caregiver responses to question 92 are coded to indicate no seizure activity, seizure activity with no definitive diagnosis of epilepsy, and seizures with a definite diagnosis of epilepsy. Using lifetime ratings, two groups of families were defined: those in which no seizure activity was reported, and those in which seizure activity was present in at least one autism patient. In addition, question 92 allows for coding of febrile seizures. Families with only febrile seizures were classified as negative for seizure activity and not included in the seizure subset analysis. Both families with positive and negative history of seizure activity were included in our overall dataset. This resulted in a dataset of 41 Caucasian families with a positive history for seizures.
Molecular analyses and genotyping

The analysis of 14 GABA receptor subunit genes was performed in 39 African-American families as previously described [23]. Briefly, between three and seven intronic, UTR and synonymous coding SNPs within each gene were identified from Applied Biosystems (ABI, Foster City, CA, USA) Assay on Demand (AoD) products, resulting in 70 SNPs within 14 GABA receptor genes on four autosomes. Genes analyzed were: GABRA1, GABRA6, GABRB2, GABRG2, and GABRP from chromosome 5; GABRA2, GABRA4, GABRB1, and GABRG1 from chromosome 4; GABRB3, GABRA5, and GABRG3 from chromosome 15; and GABRR1 and GABRR2 from chromosome 6.

Additional SNPs within GABRA4 and GABRB1 were analyzed in the extended African-American $(N=54)$ and Caucasian $(N=557)$ datasets to expand the coverage of variation across this region. Thirty-five SNPs, representative of different linkage disequilibrium (LD) blocks across the two genes (20 in GABRA4 and 15 in GABRB1), were genotyped (Fig. 1). SNP's for genotyping were selected from online databases (University of California Santa Cruz http://genome.ucsc.edu and NCBI dbSNP http://www. ncbi.nlm.nih.gov/entrez/query.fcgi?db=snp) and from resequencing of exons and surrounding areas of both GABRB1 and GABRA4 genes.

SNP genotyping was performed using Taqman allelic discrimination assays (Applied Biosystems). DNA was extracted from whole blood according to established protocols [36], and $3 \mathrm{ng}$ of genomic DNA was used per reaction. Amplification was performed on GeneAmp PCR Systems 9700 thermocyclers, with cycling conditions as recommended by Applied Biosystems. Fluorescence was measured using Applied Biosystem's 7900. Genotype discrimination was conducted using ABI Prism SDS 2.1 software. Quality control, to ensure accurate genotyping, involved two different CEPH DNAs in quadruplicate on each 384 well plate, as well as the presence of samples which were replicated elsewhere in the sample list. Additionally, $\geq 95 \%$ genotyping efficiency is required.

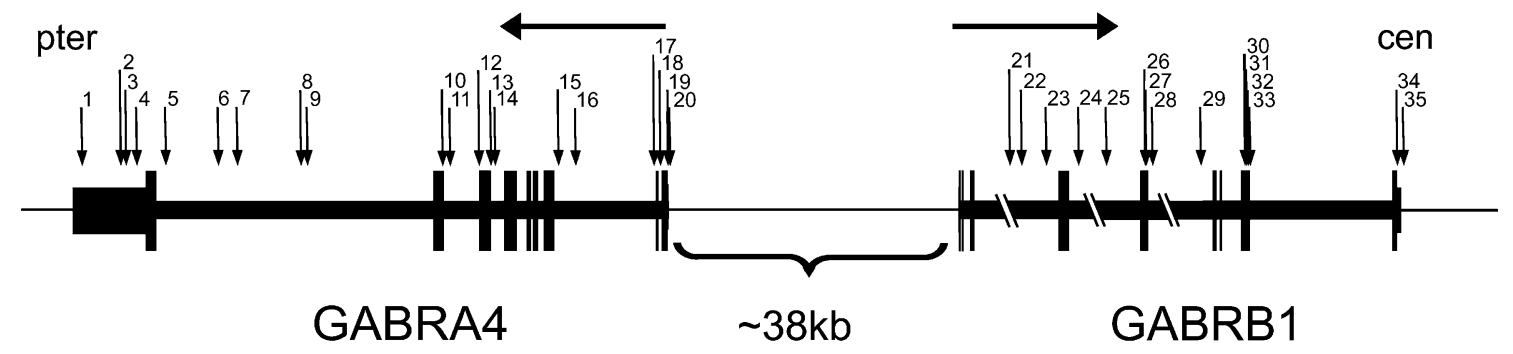
1 RS7678338
6 RS17599165
11 RS2280073
16 RS7694035
21 RS1866989
26 RS4482737
31 RS6289
2 RS6447517 7 HCV1592545
12 RS16859788
17 RS3792211
22 RS2351299
27 HCV11353524
32 RS6290
3 RS17599102
13 RS17599416
18 RS2229940
23 RS10016388
28 RS3775534
33 4P0413
4 RS7660336
9 RS1912960
14 RS3792208
19 RS13151759
24 RS1372496
29 HCV2119841
34 RS10028945
5 RS1512136 10 RS2055943
15 RS10517174
20 RS13151769 25 RS3114084
30 RS6287
35 RS3832300

Fig. 1 Approximate locations of SNPs for extended analysis within GABRA4 and GABRB1. Horizontal arrows represent transcriptional direction. Vertical arrows represent SNPs, as numbered and identified below the diagram. Pter indicates the P-terminal end of the chromosome and cen indicates the centromere 
Statistical analysis

To ensure genotyping quality, Pedcheck was run for detection of Mendelian inheritance inconsistency. One affected and one unaffected individual from each family were selected randomly for tests of Hardy-Weinberg equilibrium (HWE), which was assessed using exact tests from the Genetic Data Analysis program [37]. Pairwise LD between markers was calculated using graphical overview of linkage disequilibrium (GOLD) [38] in the parents of autism cases for both the African-American and Caucasian samples. LD was evaluated in parents to increase the available sample size for analysis and comparison between the two ethnic groups. The pedigree disequilibrium test (PDT) and its extension the genotypic pedigree disequilibrium test (genoPDT) [39, 40] were used to test for association to autism susceptibility.

The EMDR [23, 41], an extension of the MDR [42, 43], was used to test for potential gene-gene interaction, to identify specific locus combinations of interest for further investigation and validation of previous results. EMDR analysis was performed using seven SNPs, the four in $G A B R A 4$ found to show significant allelic or genotypic association in the Caucasian sample-set, and the three in $G A B R B 1$ found to be significant in the seizure subgroup. One-, two-, and three-way analysis was performed on the Caucasian dataset. For case-control pairs used in EMDR, the proband (or most completely genotyped affected child) from each multiplex and triad family was selected $(n=470$ total) as a case. Controls were generated using the untransmitted alleles from parental genotypes. In this study, a cross-validation option was not utilized. The sample size of the African-American dataset is too small to provide power for EMDR analysis, not allowing us to test for validation of the interaction seen in the paper of $\mathrm{Ma}$ et al. within the African-American dataset.

The haplotype family-based association test (HBAT; [44]) was used for haplotype association analysis using the significant SNPs in GABRA4.

\section{Results}

Allelic association studies of 70 SNPs across the 14 GABA receptor subunit genes in the 39 African-American screen set of families, revealed association in $\mathrm{rs} 2280073$ (GABRA4; $p=0.0053)$ and hcv2119841 (GABRB1; $p=0.0343$ ), the same two genes identified through allelic association and interaction analysis in the Caucasian dataset [23]. Genotypic association analysis revealed the same GABRA4 SNP, rs2280073 ( $p=0.0262)$, and marginal significance within $G A B R P, \operatorname{rs} 1862242(p=0.0471)$. The remaining SNPs showed no significant association (data not shown).

Analysis of the screening SNPs and newly identified SNPs within GABRA4 and GABRB1 in the Caucasian population $(N=557)$, and within the extended AfricanAmerican population $(N=54)$ (Table 1$)$, revealed new SNPs

Table 1 Analysis of GABRA4 and GABRBI in extended Caucasian and African-American datasets

\begin{tabular}{|c|c|c|c|c|c|c|c|c|c|}
\hline \multicolumn{5}{|l|}{ GABRA4 } & \multicolumn{5}{|l|}{ GABRB1 } \\
\hline \multirow[t]{2}{*}{ SNP } & \multicolumn{2}{|l|}{ Caucasian } & \multicolumn{2}{|c|}{ African-American } & \multirow[t]{2}{*}{ SNP } & \multicolumn{2}{|c|}{ Caucasian } & \multicolumn{2}{|c|}{ African-American } \\
\hline & $\mathrm{PDT}^{\mathrm{a}}$ & Geno $\mathrm{PDT}^{\mathrm{a}}$ & $\mathrm{PDT}^{\mathrm{a}}$ & Geno $\mathrm{PDT}^{\mathrm{a}}$ & & PDT & Geno PDT & PDT & Geno PDT \\
\hline RS7678338 & 0.9350 & 0.9923 & 0.2230 & 0.4190 & RS1866989 & 0.3071 & 0.3860 & 0.5553 & 0.7892 \\
\hline RS6447517 & 0.8826 & 0.7034 & 0.505 & 0.3930 & RS2351299 & 0.4529 & 0.0822 & 0.5775 & 0.8614 \\
\hline RS17599102 & 0.8055 & 0.8913 & 0.7518 & 0.8805 & RS10016388 & 0.1585 & 0.2660 & 0.2367 & 0.4259 \\
\hline RS17599165 & $0.0015(T)$ & $0.0009(T / T)$ & 0.6547 & 0.4304 & RS4482737 & 0.1495 & 0.2504 & 1.0000 & 1.0000 \\
\hline HCV1592545 & 0.7798 & 0.9427 & 0.2230 & 0.5313 & HCV11353524 & 0.1959 & 0.3117 & 1.0000 & 1.0000 \\
\hline RS7685553 & 1.0000 & 0.8419 & 0.4913 & 0.6044 & RS3775534 & 0.1893 & 0.1831 & 0.4913 & 0.4913 \\
\hline RS1912960 & $0.0073(\mathrm{C})$ & $0.0046(C / C)$ & 0.4111 & 0.5110 & HCV2119841 & 0.3352 & 0.0838 & 0.2278 & 0.4111 \\
\hline RS2055943 & 0.9671 & 0.9434 & 0.4927 & 0.7607 & RS6287 & 0.4045 & 0.3571 & 0.6171 & 0.6984 \\
\hline RS3792208 & 1.0000 & 0.4980 & 0.1797 & 0.1797 & RS10028945 & 0.3272 & 0.4584 & 1.0000 & 0.8179 \\
\hline RS10517174 & 0.9484 & 0.0894 & 0.7150 & 0.8903 & RS3832300 & 0.4094 & 0.6352 & 0.6547 & 0.6547 \\
\hline RS7694035 & 0.4337 & 0.6266 & 0.3657 & 0.3657 & & & & & \\
\hline RS3792211 & 0.9057 & 0.8382 & 0.6547 & 0.2895 & & & & & \\
\hline RS2229940 & 0.7873 & 0.9375 & 0.5485 & 0.7866 & & & & & \\
\hline RS13151759 & 0.7529 & 0.9326 & 0.2367 & 0.5759 & & & & & \\
\hline RS13151769 & 0.4740 & 0.7436 & 0.1824 & 0.4768 & & & & & \\
\hline
\end{tabular}

Bold indicates significant values

${ }^{a}$ Associated allele/genotype shown in parenthesis 
Table 2 Minor allele frequencies and linkage disequilibrium in Caucasian and African-American datasets

\begin{tabular}{|c|c|c|c|c|c|c|c|c|c|c|c|c|c|}
\hline SNP & Caucasian & $\begin{array}{l}\mathbf{F}^{\mathrm{a}} \\
\text { African } \\
\text { American }\end{array}$ & & 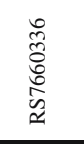 & 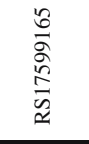 & 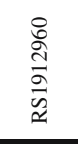 & 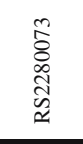 & $\begin{array}{l}\infty \\
\infty \\
\hat{0} \\
\infty \\
0 \\
\approx \\
\simeq\end{array}$ & 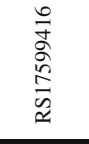 & 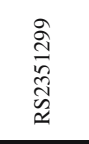 & 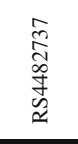 & $\begin{array}{l}\stackrel{8}{0} \\
\tilde{N} \\
\infty \\
\tilde{\hat{n}} \\
\approx\end{array}$ & \\
\hline RS7660336 & 0.500 & 0.418 & & & 0.102 & 0.286 & 0.907 & .001 & 0.104 & 0 & 0.001 & 0.009 & \\
\hline RS17599165 & 0.085 & 0.073 & $\mathrm{~N}_{-1}$ & 0.098 & & 0.331 & 0.097 & 0 & 0.853 & 0.008 & 0 & 0 & \\
\hline RS1912960 & 0.228 & 0.217 & Е & 0.376 & 0.312 & & 0.301 & 0 & 0.320 & 0.003 & 0 & 0 & \\
\hline RS2280073 & 0.500 & 0.410 & 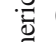 & 0.905 & 0.06 & 0.342 & & 0 & 0.113 & 0 & 0.001 & 0.008 & \\
\hline RS16859788 & 0.001 & 0.240 & $\underline{\Xi}$ & 0.236 & 0.022 & 0.085 & 0.223 & & 0 & 0 & 0 & 0 & \\
\hline RS17599416 & 0.100 & 0.060 & $\Xi$ & 0.096 & 0.709 & 0.301 & 0.104 & 0.022 & & 0.012 & 0 & 0 & \\
\hline RS2351299 & 0.178 & 0.275 & $\stackrel{\mathscr{U}}{\Xi}$ & 0.09 & 0.011 & 0.006 & 0.114 & 0.064 & 0.007 & & 0.002 & 0 & \\
\hline RS4482737 & 0.012 & 0.041 & 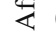 & 0.003 & 0.004 & 0 & 0.008 & 0.013 & 0.002 & 0.057 & & 0.04 & \\
\hline RS3832300 & 0.044 & 0.061 & & 0.019 & 0.002 & 0 & 0.019 & 0 & 0.005 & 0.018 & 0.019 & & \\
\hline
\end{tabular}

Bold indicates significant values

${ }^{\mathrm{a}} M A F$ minor allele frequency

with significant association. In the Caucasian dataset, rs1912960, also significant in the study of Ma et al. $(p=0.012)$, showed significant allelic association $(p=0.0073)$. Additional significant SNPs were identified in $G A B R A 4$ as well, rs17599165 $(p=0.0015)$ and $r s 1759$ $9416(p=0.0040)$. Genotypic association was also seen in these SNPs ( $p=0.0046,0.0009$, and 0.0043, respectively), as well as in a fourth SNP, also in GABRA4 (rs7660336, $p=0.0368)$. In the African-American dataset, rs2280073 $(p=0.0287)$, identified in the smaller African-American dataset above, and rs16859788 ( $p=0.0253)$, were found to be associated with the allele based test. Genotypic association was also identified in $\operatorname{rs} 16859788(p=0.0412)$. No SNPs within $G A B R B 1$ were found to be associated with autism in either ethnic group.

The majority of pairwise $r^{2}$ values between the significant SNPs were less than 0.3, in both ethnic groups (Table 2). However, a few SNPs have values between 0.3 and 0.35. SNPs, rs17599165, and rs17599416 have $r^{2}$ values of 0.709 in African-Americans and 0.853 in Caucasians, and rs7660336 and rs2280073 have a pairwise $r^{2}$ of 0.907 in Caucasians and 0.905 in African-Americans. Allele frequencies are similar, yet not identical between the two groups. One SNP, however, did have large differences in minor allele frequencies (MAFs) between the two groups, with a MAF of 0.24 in African-Americans but only 0.001 in Caucasians (Table 2). Haplotype analysis, using the four SNPs with significant allelic or genotypic association in the Caucasian families, revealed a significant global test $(p=0.014)$ in the Caucasian population, further supporting the involvement of these SNPs or another variant on the haplotype background. The haplotype composed of all risk alleles trends towards significance with autism as a risk haplotype $(p=0.0763)$, while the haplotype of all protective alleles resulted in being highly significantly protective $(p=0.0006$; Table 3$)$

Subsetting of the GABRA4 and GABRB1 data to analyze all families with positive history for seizures revealed no association to GABRA4. However, three SNPs within $G A B R B 1$ were found to be both allelically and genotypically associated with autism: $r 2351299(p=0.0163$ and $p=0.0189$ for PDT and genoPDT, respectively), rs4482737 $(p=0.0339$ and $p=0.0339)$, and $\operatorname{rs} 3832300(p=0.0253$ and $p=0.0357)$. These three SNPs all had pairwise $r^{2}$ values less than 0.1 (data not shown).

In the Caucasian population, EMDR verified the single locus effect identified through PDT analysis in rs1912960 $(p=0.024)$, and identified two different significant twolocus gene-gene effects between GABRA4 and GABRB1, rs1912960 with rs2351299 $(p=0.004)$, and rs17599416 with rs2351299 $(p=0.014)$. Several three locus effects were also significant [rs7660336, rs1912960, and rs2351 $299(p=0.012) ; \quad r s 17599165$, rs 1912960, and rs2351 $299(p=0.012) ; \mathrm{rs} 1912960, \mathrm{rs} 17599416$, and $\mathrm{rs} 2351299$ $(p=0.038)$; and rs7660336, rs17599416, and rs2351299 $(p=0.047)]$ (Table 4).

Table 3 Haplotype frequency and association in Caucasian population

\begin{tabular}{llllll}
\hline RS7660336 & RS17599165 & RS1912960 & RS17599416 & Haplotype frequency & $P$-value \\
\hline G & T & C & A & 0.503 & 0.0763 \\
C & T & C & A & 0.277 & 0.9214 \\
C & T & G & A & 0.124 & 0.6094 \\
C & A & G & G & 0.082 & $\mathbf{0 . 0 0 0 6}$ \\
\hline
\end{tabular}

Bold indicates significant values 
Table 4 EMDR results in Caucasian dataset between GABRA4 and GABRB1

\begin{tabular}{llllll}
\hline Input SNPs & & \multicolumn{3}{c}{ Significant interactions } \\
\cline { 5 - 6 } Gene & SNP number & SNP & & SNPs & $P$-values \\
\hline GABRA4 & 1 & RS7660336 & One-way & 3 & 0.024 \\
& 2 & RS17599165 & Two-way & 3,5 & 0.004 \\
& 3 & RS1912960 & & 4,5 & 0.014 \\
GABRB1 & 4 & RS17599416 & Three-way & $1,3,5$ & 0.012 \\
& 5 & RS2351299 & & $2,3,5$ & 0.012 \\
& 6 & RS4482737 & $3,4,5$ & 0.038 \\
\end{tabular}

\section{Discussion}

We have confirmed the involvement of GABRA4 in autism through identification of significantly associated SNPs within an independent African-American population. Furthermore, we have strengthened our original findings, including identification of additional associated SNPs and a significant interaction between GABRA4 and GABRB1 and in an extended dataset $(N=557)$ of Caucasian autism families. The AA dataset contains only 54 families, and in general power can be a problem in small sample sets if an effect is not seen. However, for the GABRA4 gene, we did find significant results in the AA dataset. Furthermore, studies in small datasets such as the AA dataset can also be less robust than in larger datasets. However, the fact that we find the same gene, GABRA4, significant in two different ethnic groups is supportive of the role of GABRA4 in autism risk.

The identification of two different two-way interactions between GABRA4 and GABRB1 provides additional evidence of the complex interaction of these two genes in autism. The rs1912960 (GABRA4) with rs2351299 (GABRB1) interaction is between the same two SNPs previously reported in the study of $\mathrm{Ma}$ et al., and is still significant in our larger dataset. A second significant twoway interaction was found, also including rs2351299 in GABRB1 as well as rs17599416 in GABRA4, further supporting that interactions between these two genes are involved in autism risk. Although rs 2351299 does not have a significant PDT result, it is found in both significant interaction pairs, and in each of the significant three-way interactions. The MDR approach used in these analyses is specifically designed to detect interaction effects both in the presence and absence of main effects.

It is possible that both of the two-way interactions are being identified due to LD between the two GABRA4 SNPs (rs1912960 and rs17599416). There is significant correlation between the two SNPs, although the $r^{2}$ value (Table 2) between them is not large $(0.32)$. Given that these do not appear to be causative variants, it is likely that the true variant, yet to be identified, is in LD with these GABRA4 SNPs. Examination of interaction in the independent dataset of African-American families was not possible due to the limited sample size.

We also identified variants within $G A B R B 1$ as associated within the autistic population with seizures. One of these variants, rs2351299, also shows a significant interaction with SNPs in GABRA4 (Table 4). While no effect was seen in GABRA4 for the seizure subset, the sample size may be too small to conclusively determine its role in seizure status in autism. However, the enhanced findings in GABRB1 in the seizure subset implicate $G A B R B 1$ as a contributor to genetic risk in these patients.

Despite the identification of GABRA4 in both ethnic groups, different SNPs were found to be associated. The identification of distinct SNPs within these populations may indicate differences in allele frequency and linkage disequilibrium within the two racial groups, differences in the haplotypic background in which identical causative variations originated, or differences in the causative variation. SNP rs16859788 for example, which is significant in the African-American group, shows little variation in the Caucasian dataset, therefore, providing no power for detection of an effect in this group. Other SNPs, however, show similar allele frequencies between the two populations.

Some differences in LD do exist between the two ethnic groups as well; however, the majority of the differences are small. The largest differences in LD are in pairwise values with rs16859788, which appear to mostly be due to the fact that the SNP is practically monoallelic in the Caucasian population. The Caucasian dataset suggests that there is a significant association of SNP haplotypes with autism risk, while the African-American set does not. However, this difference may be due to the small size of the AfricanAmerican dataset. Therefore, while it appears that minor allele frequency differences can account for the lack of association of rs16859788 in the Caucasian dataset, additional studies are needed to determine whether the other differences in results between the two ethnic groups are due to sample size differences, differential LD with the causative variation in the two populations, or are population-specific risk alleles.

While we have identified several associated SNPs, we do not predict any of the ones in GABRA4 to have functional consequences; therefore, it is unlikely that these are primary variants leading to the autism susceptibility. One of the SNPs identified in GABRB1 in the seizure subset, however, is in the $3^{\prime}$ untranslated region (UTR). Given that multiple GABA receptor subunits combine in varying combinations to form a functional GABA receptor, even minor changes in levels of a particular subunit may alter the makeup of receptors within a particular cell type, and alter 
the GABAergic signaling. Therefore, variations within potential regulatory regions, such as untranslated regions and promoters, could play an important role. It will be important to look at potential changes that may result from this and other potential GABRBI UTR variations, as well as sequence coding and potential regulatory regions to identify the primary variation, or variations leading to altered autism susceptibility.

In summary, these data show that the GABA receptors are implicated in the etiology of autism in two different ethnic populations and suggest seizures as a stratifying phenotype. Furthermore, these results support our earlier findings, indicating GABRA4 and GABRBI as genes contributing to autism susceptibility, independently, in the case of GABRA4, and through complex interactions with each other.

Acknowledgements We thank the patients with autism, and family members who agreed to participate in this study, as well as the personnel of the CHG at DUMC, for their input on this project. We also thank Dr. Robert Delong for referring patients and their families to the study. This research was supported in part by the National Institutes of Health (NIH) program project grant NS26630, and grants R01 NS36768, R01 AG20135, and R01 NS42165; by the National Alliance of Autism Research (NAAR); and by a gift from the Hussman Foundation. The research conducted in this study complies with current US laws. We also gratefully acknowledge the resources provided by the AGRE consortium and the participating Autism Genetic Resource Exchange (AGRE) families. The AGRE is a program of Cure Autism Now (CAN). This work used the core resources of the GCRC (MO1 RR-00095) and the CHGR at VUMC, and the $\mathrm{CHG}$ at DUMC.

\section{References}

1. Fombonne E (2002) Epidemiological trends in rates of autism. Mol Psychiatry 7(Suppl 2):S4-S6

2. Williams JG, Brayne CE, Higgins JP (2005) Systematic review of prevalence studies of autism spectrum disorders. Arch Dis Child 91(1):2-5

3. Fombonne E (1999) The epidemiology of autism: a review. Psychol Med 29:769-786

4. Fombonne E (2003) Epidemiological surveys of autism and other pervasive developmental disorders: an update. J Autism Dev Disord 33:365-382

5. Smalley SL, Asarnow RF, Spence MA (1988) Autism and genetics. A decade of research. Arch Gen Psychiatry 45:953-961

6. Bailey A, Le Couteur A, Gottesman I, Bolton P, Simonoff E, Yuzda E, Rutter M (1995) Autism as a strongly genetic disorder: evidence from a British twin study. Psychol Med 25:63-77

7. Ritvo ER, Freeman BJ, Mason-Brothers A, Mo A, Ritvo AM (1985) Concordance for the syndrome of autism in 40 pairs of afflicted twins. Am J Psychiatry 142:74-77

8. Pickles A, Bolton P, Macdonald H, Bailey A, Le Couteur A, Sim CH, Rutter M (1995) Latent-class analysis of recurrence risks for complex phenotypes with selection and measurement error: a twin and family history study of autism. Am J Hum Genet 57:717-726

9. Risch N, Spiker D, Lotspeich L, Nouri N, Hinds D, Hallmayer J, Kalaydjieva L, McCague P, Dimiceli S, Pitts T, Nguyen L, Yang J, Harper C, Thorpe D, Vermeer S, Young H, Hebert J, Lin A, Ferguson J, Chiotti C, Wiese-Slater S, Rogers T, Salmon B, Nicholas P, Myers RM (1999) A genomic screen of autism: evidence for a multilocus etiology. Am J Hum Genet 65:493-507
10. Hussman JP (2001) Suppressed GABAergic inhibition as a common factor in suspected etiologies of autism. J Autism Dev Disord 31:247-248

11. Rolf LH, Haarmann FY, Grotemeyer KH, Kehrer H (1993) Serotonin and amino acid content in platelets of autistic children. Acta Psychiatr Scand 87:312-316

12. Dhossche D, Applegate H, Abraham A, Maertens P, Bland L, Bencsath A, Martinez J (2002) Elevated plasma gammaaminobutyric acid (GABA) levels in autistic youngsters: stimulus for a GABA hypothesis of autism. Med Sci Monit 8:R1-R6

13. Blatt GJ, Fitzgerald CM, Guptill JT, Booker AB, Kemper TL, Bauman ML (2001) Density and distribution of hippocampal neurotransmitter receptors in autism: an autoradiographic study. J Autism Dev Disord 31:537-543

14. Fatemi SH, Halt AR, Realmuto G, Earle J, Kist DA, Thuras P, Merz A (2002) Purkinje cell size is reduced in cerebellum of patients with autism. Cell Mol Neurobiol 22:171-175

15. Bauman ML, Kemper TL (2005) Neuroanatomic observations of the brain in autism: a review and future directions. Int $\mathrm{J}$ Dev Neurosci 23:183-187

16. Bundey S, Hardy C, Vickers S, Kilpatrick MW, Corbett JA (1994) Duplication of the 15q11-13 region in a patient with autism, epilepsy and ataxia. Dev Med Child Neurol 36:736-742

17. Wolpert CM, Menold MM, Bass MP, Qumsiyeh MB, Donnelly SL, Ravan SA, Vance JM, Gilbert JR, Abramson RK, Wright $\mathrm{HH}$, Cuccaro ML, Pericak-Vance MA (2000) Three probands with autistic disorder and isodicentric chromosome 15. Am J Med Genet 96:365-372

18. Maestrini E, Lai C, Marlow A, Matthews N, Wallace S, Bailey A, Cook EH, Weeks DE, Monaco AP (1999) Serotonin transporter (5-HTT) and gamma-aminobutyric acid receptor subunit beta3 (GABRB3) gene polymorphisms are not associated with autism in the IMGSA families. The international molecular genetic study of autism consortium. Am J Med Genet 88:492-496

19. Shao Y, Cuccaro ML, Hauser ER, Raiford KL, Menold MM, Wolpert CM, Ravan SA, Elston L, Decena K, Donnelly SL, Abramson RK, Wright HH, DeLong GR, Gilbert JR, PericakVance MA (2003) Fine mapping of autistic disorder to chromosome $15 \mathrm{q} 11-\mathrm{q} 13$ by use of phenotypic subtypes. Am J Hum Genet 72:539-548

20. Buxbaum JD, Silverman JM, Smith CJ, Greenberg DA, Kilifarski M, Reichert J, Cook EH Jr, Fang Y, Song CY, Vitale R (2002) Association between a GABRB3 polymorphism and autism. Mol Psychiatry 7:311-316

21. Nurmi EL, Bradford Y, Chen Y, Hall J, Arnone B, Gardiner MB, Hutcheson HB, Gilbert JR, Pericak-Vance MA, CopelandYates SA, Michaelis RC, Wassink TH, Santangelo SL, Sheffield VC, Piven J, Folstein SE, Haines JL, Sutcliffe JS (2001) Linkage disequilibrium at the Angelman syndrome gene UBE3A in autism families. Genomics 77:105-113

22. Slopien A, Rajewski A, Budny B, Czerski P (2002) Evaluation of q11-q13 locus of chromosome 15 aberrations and polymorphisms in the B3 subunit of the GABA-A receptor gene (GABRB3) in autistic patients. Psychiatr Pol 36:779-791

23. Ma DQ, Whitehead PL, Menold MM, Martin ER, Ashley-Koch AE, Mei H, Ritchie MD, DeLong GR, Abramson RK, Wright HH, Cuccaro ML, Hussman JP, Gilbert JR, Pericak-Vance MA (2005) Identification of significant association and gene-gene interaction of GABA receptor subunit genes in autism. Am J Hum Genet 77:377-388

24. Macdonald RL, Gallagher MJ, Feng HJ, Kang J (2004) GABA (A) receptor epilepsy mutations. Biochem Pharmacol 68: $1497-1506$

25. Fombonne E (2003) The prevalence of autism. JAMA 289:87-89

26. Yeargin-Allsopp M, Rice C, Karapurkar T, Doernberg N, Boyle C, Murphy C (2003) Prevalence of autism in a US metropolitan area. JAMA 289:49-55 
27. Cuccaro ML, Donnelly S, Cope H, Wolpert C, Carney R, Abramson RK, Hall A, Wright HH, Gilbert JR, Pericak-Vance MA (2005) Autism in African-American (AA) families: phenotypic findings. American Society of Human Genetics 55th Annual Meeting, Salt Lake City, UT

28. American Psychiatric Association (1994) In: Frances ATFC (ed) Diagnostic and statistical manual of mental disorders (DSM-IV), vol IV. American Psychiatric Press, Washington, DC

29. Rutter M, LeCouteur A, Lord C (2003) Autism diagnostic interview, revised (ADI-R). Western Psychological Services, Los Angeles, CA

30. Lord C, Rutter M, DiLavore P, Risi S (1999) Autism diagnostic observation schedule-WPS (WPS edn). Western Psychological Association, Los Angeles

31. Sparrow SS, Balla D, Cicchetti D (1984) Vineland adaptive behavior scales, interview edn. AGS Publishing, Circle Pines, MN

32. Wechsler D (2003) Wechsler intelligence scale for children (WISC-IV), 4th edn. Psychological Corporation, San Antonio, Texas

33. Elliott CD, Murray GJ, Pearson LS (1990) The differential ability scales. San Antonio, Texas

34. Mullen EM (1995) Mullen scales of early learning, AGS edn. American Guidance Service, Circle Pines, MN

35. Roid GH, Miller LJ (1997) Leiter international performance scale, revised. Stoeling, Woodale, IL

36. Vance JM (1998) The collection of biological samples for DNA analysis. In: Haines JL, Pericak-Vance MA (eds) Approaches to gene mapping in complex human diseases. Wiley, New York
37. Zaykin D, Zhivotovsky L, Weir BS (1995) Exact tests for association between alleles at arbitrary numbers of loci. Genetica 96:169-178

38. Abecasis GR, Cookson WO (2000) GOLD-graphical overview of linkage disequilibrium. Bioinformatics 16:182-183

39. Martin ER, Monks SA, Warren LL, Kaplan NL (2000) A test for linkage and association in general pedigrees: the pedigree disequilibrium test. Am J Hum Genet 67:146-154

40. Martin ER, Bass MP, Gilbert JR, Pericak-Vance MA, Hauser ER (2003) Genotype-based association test for general pedigrees: the genotype-PDT. Genet Epidemiol 25:203-213

41. Mei H, Ma DQ, Ashley-Koch A, Martin ER (2005) Extension of multifactor dimensionality reduction for identifying multilocus effects in the GAW14 simulated data. BMC Genet 6 (Suppl 1)

42. Ritchie MD, Hahn LW, Roodi N, Bailey LR, Dupont WD, Parl FF, Moore JH (2001) Multifactor-dimensionality reduction reveals high-order interactions among estrogen-metabolism genes in sporadic breast cancer. Am J Hum Genet 69:138-147

43. Hahn LW, Ritchie MD, Moore JH (2003) Multifactor dimensionality reduction software for detecting gene-gene and geneenvironment interactions. Bioinformatics 19:376-382

44. Horvath S, Xu X, Lake SL, Silverman EK, Weiss ST, Laird NM (2004) Family-based tests for associating haplotypes with general phenotype data: application to asthma genetics. Genet Epidemiol 26:61-69 